\title{
Robust Synthesis for Master-Slave Synchronization of Lur'e Systems
}

\author{
Johan A. K. Suykens, Paul F. Curran, and Leon O. Chua
}

\begin{abstract}
In this paper a method for robust synthesis of full static-state error feedback and dynamic-output error feedback for master-slave synchronization of Lur'e systems is presented. Parameter mismatch between the systems is considered in the synchronization schemes. Sufficient conditions for uniform synchronization with a bound on the synchronization error are derived, based on a quadratic Lyapunov function. The matrix inequalities from the case without parameter mismatch between the Lur'e systems remain preserved, but an additional robustness criterion must be taken into account. The robustness criterion is based on an uncertainty relation between the synchronization error bound and the parameter mismatch. The robust synthesis method is illustrated on Chua's circuit with the double scroll. One observes that it is possible to synchronize the master-slave systems up to a relatively small error bound, even in the case of different qualitative behavior between the master and the uncontrolled slave system, such as limit cycles and stable equilibria.
\end{abstract}

Index Terms - Chua's circuit, Lur'e systems, matrix inequalities, synchronization.

\section{INTRODUCTION}

$\mathbf{M}$ ASTER-SLAVE synchronization schemes for Lur'e systems have been studied in [5], [6], and [16] for the autonomous case with static-state feedback or dynamicoutput feedback applied to the slave system. With respect to secure communications applications [8], the nonautonomous case has been studied in [17] and [18] where an informationcarrying message signal is considered as an external input. In these works the synchronization problem has been approached from the viewpoint of control theory. The autonomous case has been studied with respect to absolute stability theory with sufficient conditions for global asymptotic stability of the error system, either based on quadratic or Lur'e-Postnikov Lyapunov functions. For the nonautonomous case, the synchronization scheme has been interpreted as a model-reference control scheme in standard plant form, with exogenous input and regulated output, according to modern control theory [1], [11]. In this way, additive channel noise has been taken into account in the design procedure.

Manuscript received September 12, 1996; revised June 8, 1998. This work was supported in part by the Office of Naval Research under Grant N0001496-1-0753 and in part by the Fulbright Fellowship Program. This paper was recommended by Associate Editor U. Helmke.

J. A. K. Suykens is with the Katholieke Universiteit Leuven, Department of Electrical Engineering, ESAT-SISTA, B-3001 Leuven, Belgium.

P. F. Curran is with Electronic and Electrical Engineering, University College, Belfield, Dublin 4, Ireland.

L. O. Chua is with the Department of Electrical Engineering and Computer Science, University of California at Berkeley, Berkeley, CA 94720 USA.

Publisher Item Identifier S 1057-7122(99)05575-0.
However, these synchronization schemes assume that the master-slave Lur'e systems are identical. In this paper we study the influence of parameter mismatch between the Lur'e systems. Previous work on robust synchronization has been reported in [21], [5], and [19]. According to [21], the case of nonidentical Lur'e systems requires the definition of a synchronization error bound because zero synchronization error cannot be achieved. In this paper we present matrix inequalities [2] for the synchronization schemes with static-state feedback or dynamic-output feedback. The matrix inequalities give sufficient conditions for uniform synchronization with a certain error bound. The same form is preserved for the matrix inequalities as for the case of identical master-slave Lur'e systems. Uncertainty relations between the bound on the synchronization error and the parameter mismatch follow from the derived theorems. The additive perturbation, which is related to the parameter mismatch and is considered with respect to the nominal identical Lur'e systems, is assumed to be unstructured [11]. Finally, a robust synthesis method is presented, which involves solving a nonlinear optimization problem. This optimization problem is based on the matrix inequality and the robustness criterion, where the latter follows from the uncertainty relation. The robust synthesis method is illustrated on Chua's circuit. From the simulation results one observes that a relatively large parameter mismatch can be allowed such that the systems remain synchronized with a relatively small synchronization error bound. In this sense, it is possible to synchronize the slave system, which behaves as the double scroll in the uncontrolled case, to a master system which behaves either chaotically, periodically, or has stable equilibrium points. These results have been obtained both for the case of full static-state feedback and dynamic-output feedback.

This paper is organized as follows. In Section II, we present the synchronization schemes with nonidentical Lur'e systems for the case of static-state error feedback and dynamic-output error feedback. In Section III, we describe the corresponding error systems. In Section IV, criteria for robust synchronization are derived. In Section V, the robust synthesis method is presented, which is based on the derived matrix inequalities. Finally, in Section VI the method is illustrated by applying it to Chua's circuit.

\section{SyNCHRONIZATION SCHEMES}

In this section we describe master-slave synchronization of Lur'e systems for two cases: full static-state error feedback and dynamic-output error feedback. 


\section{A. Full Static-State Error Feedback}

Consider the master-slave synchronization scheme with full static-state error feedback [21], [5], [17]

$$
\begin{aligned}
\mathcal{M}_{s}: & \dot{x}=A_{1} x+B_{1} \sigma\left(C_{1} x\right) \\
\mathcal{S}_{s}: & \dot{z}=A_{2} z+B_{2} \sigma\left(C_{2} z\right)+u_{s} \\
\mathcal{C}_{s}: & u_{s}=F(x-z)
\end{aligned}
$$

with master system $\mathcal{M}_{s}$, slave system $\mathcal{S}_{s}$, and controller $\mathcal{C}_{s}$. The index $s$ refers to the static feedback case. The master and slave system are Lur'e systems with state vectors $x, z \in \mathbb{R}^{n}$, respectively, and matrices $A_{i} \in \mathbb{R}^{n \times n}, B_{i} \in \mathbb{R}^{n \times n_{h_{h}}}$, and $C_{i} \in \mathbb{R}^{n_{h} \times n}(i=1,2)$. A Lur'e system is a linear dynamical system, feedback interconnected to a static nonlinearity $\sigma(\cdot)$ that satisfies a sector condition [9], [20] (here it has been represented as a recurrent neural network with one hidden layer, activation function $\sigma(\cdot)$, and $n_{h}$ hidden units [15]). We assume that $\sigma(\cdot): \mathbb{R}^{n_{h}} \rightarrow \mathbb{R}^{n_{h t}}$ is a diagonal and continuous nonlinearity (but possibly nondifferentiable at a countable number of points) with $\sigma_{i}(\cdot)$ belonging to sector $[0, k]$, i.e. $\sigma_{i}(\xi)\left[\sigma_{i}(\xi)-k \xi\right] \leq 0, \forall \xi \in \mathbb{R}$ for $i=1, \cdots, n_{h}$. The scheme aims at synchronizing the master system to the slave system by applying full static-state error feedback to the slave system using the control signal $u_{s} \in \mathbb{R}^{n}$ with feedback matrix $F \in \mathbb{R}^{n \times n}$.

The Lur'e systems are assumed to be nonidentical. The parameter mismatch between the systems, $\left\|A_{1}-A_{2}\right\|_{2}, \| B_{1}-$ $B_{2}\left\|_{2},\right\| C_{1}-C_{2} \|_{2}$ is assumed to be relatively small. The aim of this paper is precisely to design the feedback matrix $F$ such that the scheme is robust with respect to this parameter mismatch.

\section{B. Dynamic-Output Error Feedback}

In addition to the state feedback scheme we will also study the dynamic-output error feedback scheme introduced in [16]

$$
\begin{aligned}
\mathcal{M}_{d}: & \left\{\begin{array}{l}
\dot{x}=A_{1} x+B_{1} \sigma\left(C_{1} x\right) \\
p=H x
\end{array}\right. \\
\mathcal{S}_{d}: & \left\{\begin{array}{l}
\dot{z}=A_{2} z+B_{2} \sigma\left(C_{2} z\right)+D u_{d} \\
q=H z
\end{array}\right. \\
\mathcal{C}_{d}: & \left\{\begin{array}{l}
\dot{\rho}=E \rho+G(p-q) \\
u_{d}=M \rho+N(p-q)
\end{array}\right.
\end{aligned}
$$

with master system $\mathcal{M}_{d}$, slave system $\mathcal{S}_{d}$, and a controller $\mathcal{C}_{d}$. The index $d$ refers to the dynamic feedback case. For the master and slave system we consider the state vectors, system matrices, and nonlinearity as described for (1). The output vectors of the master and slave system are $p, q \in \mathbb{R}^{l}$ with $l \leq n$. The slave system is controlled by means of the control vector $u_{d} \in \mathbb{R}^{m}$ through the matrix $D \in \mathbb{R}^{n \times m}$. The signal $u_{d}$ is the output of a linear dynamic-output feedback controller. The input of this controller is the output error $p-q$. The linear dynamic controller has state vector $\rho \in \mathbb{R}^{n_{c}}$ and consists of the matrices $E \in \mathbb{R}^{n_{c} \times n_{c}}, G \in \mathbb{R}^{n_{c} \times l}, M \in \mathbb{R}^{m \times n_{c}}$, and $N \in \mathbb{R}^{m \times l}$.

In [16] this scheme has been studied for identical Lur'e systems. As for the state feedback case, we are interested here in designing the controller $\mathcal{C}_{d}$ such that a high robustness is obtained with respect to parameter mismatch between the Lur'e systems.

\section{ERROR SYSTEMS}

Defining the error signal as $e=x-z$, the aim of the synchronization schemes is to design the controllers $\mathcal{C}_{s}, \mathcal{C}_{d}$ such that $\|e(t)\|_{2} \rightarrow 0$ as $t \rightarrow \infty$. However, a zero error is only obtainable in the case of identical master-slave systems. For the case of parameter mismatch between the systems, an error bound must be considered instead. This will be further discussed in the following section.

Denoting the state feedback synchronization scheme as

$$
\begin{aligned}
\dot{x} & =f_{s}(x) \\
\dot{z} & =g_{s}(z, x)
\end{aligned}
$$

with continuous nonlinear mappings $f_{s}(\cdot): \mathbb{R}^{n} \rightarrow \mathbb{R}^{n}$, $g_{s}(\cdot, \cdot): \mathbb{R}^{n} \times \mathbb{R}^{n} \rightarrow \mathbb{R}^{n}$ one obtains the error system

$$
\mathcal{E}_{s}: \dot{e}=f_{s}(x)-g_{s}(z, x) \text {. }
$$

Inspired by the proof of Theorem 14 in [21], we make the following decomposition for the error system:

$$
\dot{e}=v_{s}(x, z)+w_{s}(x)
$$

with

$$
\begin{aligned}
v_{s}(x, z) & =g_{s}(x, x)-g_{s}(z, x) \\
& =\left(A_{2}-F\right) e+B_{2} \eta\left(C_{2} e ; z\right) \\
w_{s}(x) & =f_{s}(x)-g_{s}(x, x) \\
& =\Delta A x+B_{1} \sigma\left(C_{1} x\right)-B_{2} \sigma\left(C_{2} x\right)
\end{aligned}
$$

where $\Delta A=A_{1}-A_{2}, \eta\left(C_{2} e ; z\right)=\sigma\left(C_{2} e+C_{2} z\right)-\sigma\left(C_{2} z\right)$.

Denoting the dynamic synchronization scheme as

$$
\begin{aligned}
& \dot{x}=f_{d}(x) \\
& \dot{z}=g_{d}(z, x, \rho) \\
& \dot{\rho}=h_{d}(\rho, x, z)
\end{aligned}
$$

with continuous nonlinear mappings $f_{d}(\cdot): \mathbb{R}^{n} \rightarrow \mathbb{R}^{n}$, $g_{d}(\cdot, \cdot, \cdot): \mathbb{R}^{n} \times \mathbb{R}^{n} \times \mathbb{R}^{n_{c}} \rightarrow \mathbb{R}^{n}, h_{d}(\cdot, \cdot, \cdot): \mathbb{R}^{n_{c}} \times \mathbb{R}^{n} \times \mathbb{R}^{n} \rightarrow$ $\mathbb{R}^{n_{c}}$, one obtains the error system

$$
\mathcal{E}_{d}: \dot{e}=f_{d}(x)-g_{d}(z, x, \rho) .
$$

We make the following decomposition:

$$
\dot{e}=v_{d}(x, z, \rho)+w_{d}(x, \rho)
$$

with

$$
\begin{aligned}
v_{d}(x, z, \rho) & =g_{d}(x, x, \rho)-g_{d}(z, x, \rho) \\
& =\left(A_{2}-D N H\right) e+B_{2} \eta\left(C_{2} e ; z\right) \\
w_{d}(x, \rho) & =f_{d}(x)-g_{d}(x, x, \rho) \\
& =\varphi(x)-D M \rho
\end{aligned}
$$

where $\Delta A=A_{1}-A_{2}, \eta\left(C_{2} e ; z\right)=\sigma\left(C_{2} e+C_{2} z\right)-\sigma\left(C_{2} z\right)$ and $\varphi(x)=\Delta A x+B_{1} \sigma\left(C_{1} x\right)-B_{2} \sigma\left(C_{2} x\right)$. 


\section{CRITERIA FOR Robust SyNCHRONIZATION}

In order to derive criteria for synchronization of the schemes (1)-(2) with parameter mismatch between the systems, we first must introduce assumptions on the nonlinearity in the error system and on the norm of the state vector of the master system.

Assumption 1: The nonlinearity $\eta\left(C_{2} e ; z\right)$ belongs to sector $[0, k]$

$$
\begin{gathered}
0 \leq \frac{\eta_{i}\left(c_{2_{i}}^{T} e ; z\right)}{c_{2_{i}}^{T} e}=\frac{\sigma_{i}\left(c_{2_{i}}^{T} e+c_{2_{i}}^{T} z\right)-\sigma\left(c_{2_{i}}^{T} z\right)}{c_{2_{i}}^{T} e} \leq k, \\
\forall e, z ; i=1, \cdots, n_{h}\left(c_{2_{i}}^{T} e \neq 0\right)
\end{gathered}
$$

where $c_{2}^{T}$ denotes the $i$ th row vector of $C_{2}$.

The following inequality holds then:

$$
\begin{aligned}
& \eta_{i}\left(c_{2_{i}}^{T} e ; z\right)\left[\eta_{i}\left(c_{2_{i}}^{T} e ; z\right)-k c_{2_{i}}^{T} e\right] \leq 0, \\
& \quad \forall e, z ; i=1, \cdots, n_{h} .
\end{aligned}
$$

It follows from the mean value theorem that for differentiable $\sigma(\cdot)$ the sector condition $[0, k]$ on $\eta(\cdot)$ corresponds to [5]

$$
0 \leq \frac{d}{d \rho} \sigma_{i}(\rho ; z) \leq k, \quad \forall \rho, z ; i=1, \cdots, n_{h} .
$$

For $w_{s}(\cdot)$ there exists a positive real constant $\beta_{s}$ such that

$$
\left\|w_{s}(x)\right\|_{2}<\beta_{s}\|x\|_{2}, \quad \forall x \in \mathbb{R}^{n} .
$$

The same holds for the function $\varphi(\cdot)$ with constant $\beta_{d}$.

Assumption 2: Master systems $\mathcal{M}_{s}$ and $\mathcal{M}_{d}$ satisfy the condition that there exists a positive real constant $\delta$ such that for any initial condition $x_{0}$ there exists time $T\left(x_{0}\right)$ for which

$$
\left\|x\left(t ; x_{0}\right)\right\|_{2} \leq \delta, \quad \forall t \geq T .
$$

This assumption is based on the work of [5]. From a practical point of view this is a reasonable assumption, in particular for chaotic Lur'e systems, because one is not interested in employing a master system that possesses unbounded trajectories.

Because the master-slave systems are nonidentical, the synchronization error will not tend asymptotically to zero. Therefore, the following definition of synchronization with error bound $\epsilon$ is employed [21].

Definition 1: The synchronization schemes (1)-(2) uniformly synchronize with error bound $\epsilon$ if there exists a $\delta_{0}>0$ and a $T \geq 0$ such that if $\|x(0)-z(0)\|_{2} \leq \delta_{0}$ then $\|x(t)-z(t)\|_{2} \leq \epsilon$ for all $t \geq T$.

For the synchronization scheme with static feedback we consider the following positive definite quadratic Lyapunov function (which is radially unbounded):

$$
V(e)=e^{T} P e, \quad P=P^{T}>0 .
$$

Theorem 1: Suppose Assumptions 1 and 2 and that there exists a diagonal positive definite matrix $\Lambda=\operatorname{diag}\left\{\lambda_{i}\right\} \in$ $\mathbb{R}^{n_{h_{t}} \times n_{h_{h}}}$, a symmetric positive definite matrix $P \in \mathbb{R}^{n \times n}$, a matrix $F \in \mathbb{R}^{n \times n}$, and a positive real constant $\alpha$ such that the matrix inequality

$$
\begin{aligned}
Y & =Y^{T} \\
& =\left[\begin{array}{cc}
\left(A_{2}-F\right)^{T} P+P\left(A_{2}-F\right)+\alpha I & P B_{2}+k C_{2}^{T} \Lambda \\
B_{2}^{T} P+k \Lambda C_{2} & -2 \Lambda
\end{array}\right] \\
& <0
\end{aligned}
$$

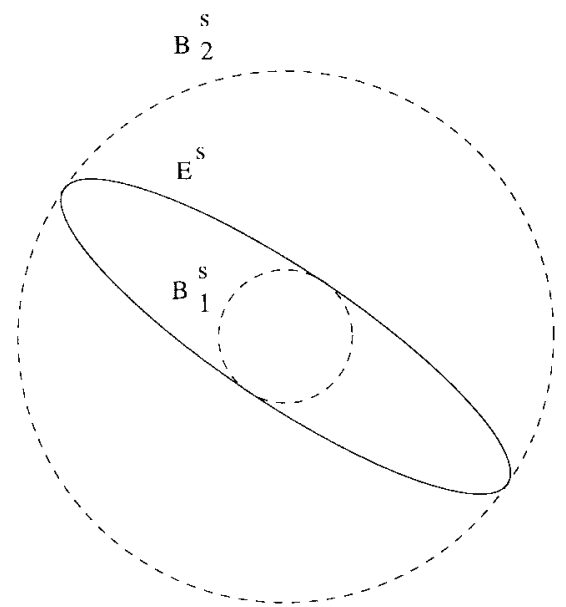

Fig. 1. Illustration of the balls $\mathcal{B}_{1}^{s}, \mathcal{B}_{2}^{s}$ and the ellipsoid $\mathcal{E}^{s}$ with respect to Theorem 1.

is satisfied. Define $\gamma=2 \beta_{s} \delta \sigma_{\max }(P)$ where $\beta_{s}, \delta$ are defined according to (12) and (13), respectively, and $\sigma_{\max }(P)$ denotes the maximal singular value of $P$. If there exist positive real constants $c, c_{2}$ such that (Fig. 1)

$$
\begin{aligned}
& \mathcal{B}_{1}^{s}=\left\{e \mid e^{T} e \leq \gamma^{2} / \alpha^{2}\right\} \subset \\
& \mathcal{E}^{s}=\left\{e \mid e^{T} P e \leq c\right\} \subset \mathcal{B}_{2}^{s}=\left\{e \mid e^{T} e \leq c_{2}\right\}
\end{aligned}
$$

then the synchronization scheme (1) is uniformly synchronizing with error bound $\sqrt{c_{2}}$.

Proof: Taking the time derivative of the Lyapunov function (14) and using the inequalities (10) one obtains

$$
\begin{aligned}
\dot{V}= & \dot{e}^{T} P e+e^{T} P \dot{e} \\
= & {\left[v_{s}(x, z)+w_{s}(x)\right]^{T} P e+e^{T} P\left[v_{s}(x, z)+w_{s}(x)\right] } \\
\leq & {\left[\left(A_{2}-F\right) e+B_{2} \eta\right]^{T} P e+e^{T} P\left[\left(A_{2}-F\right) e+B_{2} \eta\right] } \\
& +2 e^{T} P w_{s}(x)-\sum_{i} 2 \lambda_{i} \eta_{i}\left(\eta_{i}-k c_{2_{i}}^{T} e\right) \\
\leq & \zeta^{T} Y_{0} \zeta+2 \beta_{s} \delta \sigma_{\max }(P)\|e\|_{2}
\end{aligned}
$$

where $\zeta=[e ; \eta]$ and

$$
Y_{0}=\left[\begin{array}{cc}
\left(A_{2}-F\right)^{T} P+P\left(A_{2}-F\right) & P B_{2}+k C_{2}^{T} \Lambda \\
B_{2}^{T} P+k \Lambda C_{2} & -2 \Lambda
\end{array}\right] .
$$

From (15) we obtain the upper bound $\zeta^{T} Y_{0} \zeta \leq-\alpha e^{T} e$. This yields

$$
\begin{aligned}
\dot{V} & \leq-\alpha\|e\|_{2}^{2}+\gamma\|e\|_{2} \\
& =\|e\|_{2}\left[-\alpha\|e\|_{2}+\gamma\right] .
\end{aligned}
$$

The latter expression is negative if

$$
\|e(t)\|_{2}>\gamma / \alpha, \quad \forall t .
$$

When $c$ is determined such that $\mathcal{E}^{s}=\left\{e \mid e^{T} P e \leq c\right\}$ is the smallest ellipsoid containing the ball $\mathcal{B}_{1}^{s}=\left\{e \mid e^{T} e \leq\right.$ $\left.\gamma^{2} / \alpha^{2}\right\}$ the trajectory will enter the ellipsoid $\mathcal{E}^{s}$ for every initial condition $e(0)$. Note that the ellipsoid $\mathcal{E}^{s}$, which is parametrized in terms of $c$, is directly related to the level set of the Lyapunov function $V$ [20]. Hence, for every initial condition, the limit set of the error system is nonempty, closed, and bounded and belongs to $\mathcal{E}^{s}$ [20]. Therefore, the 
error system will uniformly synchronize with error bound $\sqrt{c_{2}}$ where $c_{2}$ is determined such that $\mathcal{B}_{2}^{s}$ is the smallest ball containing $\mathcal{E}^{s}$.

\section{Remarks:}

1) We stress that the condition (15), being based on a quadratic Lyapunov function, is only sufficient and possibly conservative. However, from absolute stability theory of Lur'e systems [9], [20] one knows that this matrix inequality is related to the circle criterion (by means of the KYP Lemma) and, in this sense, is meaningful. The numerical algorithms which follow this section have two tasks. First, to confirm that for chaotic Lur'e systems, such as Chua's circuit, a solution to the LMI exists and second, to explain how one can find one.

2) Convex optimization procedures for finding an outer approximation of a union of ellipsoids and an intersection of ellipsoids are discussed in [2, p. 43]. These methods can be used in order to construct $\mathcal{B}_{2}^{s}, \mathcal{E}^{s}$ given $\mathcal{B}_{1}^{s}$ and finding $c, c_{2}$. On the other hand, it follows from Fig. 1 that $\sqrt{c_{2}}=\gamma \kappa(P) / \alpha$ with $\kappa(P)=\left(\sigma_{\max }(P) / \sigma_{\min }(P)\right)$ the condition number of matrix $P$.

3) Note that for $P=I$ the synchronization scheme (1) is uniformly synchronizing with error bound $2 \beta_{s} \delta / \alpha$. In this case, the sets $\mathcal{B}_{1}^{s}, \mathcal{B}_{2}^{s}$, and $\mathcal{E}^{s}$ are identical.

For the synchronization scheme with dynamic feedback we consider the following positive definite quadratic Lyapunov function:

$$
\begin{aligned}
V(\xi) & =\xi^{T} P \xi=\left[e^{T} \rho^{T}\right]\left[\begin{array}{ll}
P_{11} & P_{12} \\
P_{21} & P_{22}
\end{array}\right]\left[\begin{array}{l}
e \\
\rho
\end{array}\right], \\
P & =P^{T}>0
\end{aligned}
$$

with $\xi=[e ; \rho]$.

Theorem 2: Suppose Assumptions 1 and 2 and that there exists a diagonal positive definite matrix $\Lambda=$ $\operatorname{diag}\left\{\lambda_{i}\right\} \in \mathbb{R}^{n_{h} \times n_{h}}$, a symmetric positive definite matrix $P \in \mathbb{R}^{\left(n+n_{c}\right) \times\left(n+n_{c}\right)}$, controller matrices $E, G, M, N$, and positive real constants $\alpha_{1}, \alpha_{2}$ such that the matrix inequality

$$
Z=Z^{T}=\left[\begin{array}{ccc}
Z_{11}+\alpha_{1} I & Z_{12} & Z_{13} \\
Z_{12}^{T} & Z_{22}+\alpha_{2} I & Z_{23} \\
Z_{13}^{T} & Z_{23}^{T} & Z_{33}
\end{array}\right]<0
$$

with

$$
\begin{aligned}
Z_{11}= & \left(A_{2}-D N H\right)^{T} P_{11}+P_{11}\left(A_{2}-D N H\right) \\
& +H^{T} G^{T} P_{21}+P_{12} G H \\
Z_{12}= & \left(A_{2}-D N H\right)^{T} P_{12}+H^{T} G^{T} P_{22}-P_{11} D M \\
& +P_{12} E \\
Z_{13}= & P_{11} B_{2}+k C_{2}^{T} \Lambda \\
Z_{22}= & E^{T} P_{22}+P_{22} E-M^{T} D^{T} P_{12}-P_{21} D M \\
Z_{23}= & P_{21} B_{2} \\
Z_{33}= & -2 \Lambda
\end{aligned}
$$

is satisfied. Define $P_{I}=\operatorname{diag}\left\{I_{n} / \eta_{1}^{2}, I_{n_{c}} / \eta_{2}^{2}\right\}$ with $\eta_{1}=$ $\left(\tau_{1} / 2 \alpha_{1}\right)+(1 / 2) \sqrt{\left(\tau_{1}^{2} / \alpha_{1}^{2}\right)+\left(\tau_{2}^{2} / \alpha_{1} \alpha_{2}\right)}, \eta_{2}=\left(\tau_{2} / 2 \alpha_{2}\right)+$ $(1 / 2) \sqrt{\left(\tau_{1}^{2} / \alpha_{1} \alpha_{2}\right)+\left(\tau_{2}^{2} / \alpha_{2}^{2}\right)}$ where $\tau_{1}=2 \beta_{d} \delta \sigma_{\max }\left(P_{11}\right)$, $\tau_{2}=2 \beta_{d} \delta \sigma_{\max }\left(P_{12}\right) . \beta_{d}, \delta$ are defined according to (12)

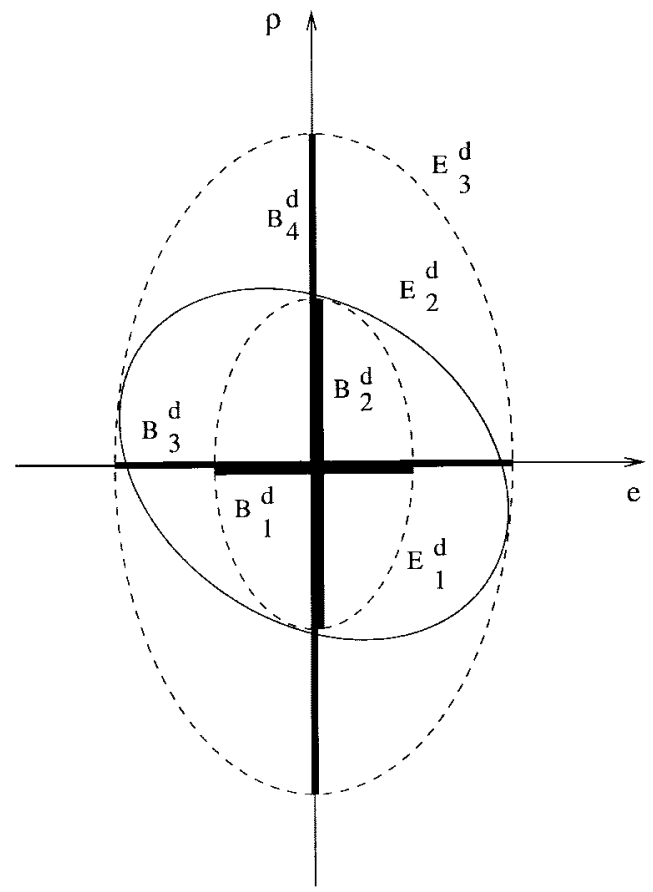

Fig. 2. Illustration of the balls $\mathcal{B}_{1}^{d}, \mathcal{B}_{2}^{d}, \mathcal{B}_{3}^{d}, \mathcal{B}_{4}^{d}$ and the ellipsoids $\mathcal{E}_{1}^{d}, \mathcal{E}_{2}^{d}$, $\mathcal{E}_{3}^{d}$ with respect to Theorem 2. A simplified case is shown for $n=1 n_{c}=1$ such that the balls correspond to line segments.

and (13), respectively. If there exist positive real constants $c_{1}, c_{2}, c_{3}, c_{4}$ such that (Fig. 2)

$$
\begin{aligned}
& \mathcal{B}_{1}^{d}=\left\{e \mid e^{T} e \leq \eta_{1}^{2}\right\} \subset \mathcal{E}_{1}^{d}=\left\{\xi \mid \xi^{T} P_{I} \xi \leq 1\right\} \\
& \mathcal{B}_{2}^{d}=\left\{\rho \mid \rho^{T} \rho \leq \eta_{2}^{2}\right\} \subset \mathcal{E}_{1}^{d} \\
& \mathcal{B}_{3}^{d}=\left\{e \mid e^{T} e \leq c_{3}\right\} \subset \mathcal{E}_{3}^{d}=\left\{\xi \mid \xi^{T} P_{I} \xi \leq c_{2}\right\} \\
& \mathcal{B}_{4}^{d}=\left\{\rho \mid \rho^{T} \rho \leq c_{4}\right\} \subset \mathcal{E}_{3}^{d}
\end{aligned}
$$

and

$$
\mathcal{E}_{1}^{d} \subset \mathcal{E}_{2}^{d}=\left\{\xi \mid \xi^{T} P \xi \leq c_{1}\right\} \subset \mathcal{E}_{3}^{d}
$$

then the synchronization scheme (2) is uniformly synchronizing with error bound $\sqrt{c_{3}}$.

Proof: Taking the time derivative of the Lyapunov function (16) and, using the inequalities (10), one obtains

$$
\begin{aligned}
\dot{V}= & \dot{\xi}^{T} P \xi+\xi^{T} P \dot{\xi} \\
= & {\left[v_{d}(x, z, \rho)+w_{d}(x, \rho)\right]^{T}\left(P_{11} e+P_{12} \rho\right) } \\
& +\dot{\rho}^{T}\left(P_{21} e+P_{22} \rho\right)+\left(e^{T} P_{11}+\rho^{T} P_{21}\right) \\
& \cdot\left[v_{d}(x, z, \rho)+w_{d}(x, \rho)\right]+\left(e^{T} P_{12}+\rho^{T} P_{22}\right) \dot{\rho} \\
\leq & {\left[\left(A_{2}-D N H\right) e+B_{2} \eta-D M \rho+\varphi(x)\right]^{T} } \\
& \cdot\left(P_{11} e+P_{12} \rho\right)+(E \rho+G H e)^{T}\left(P_{21} e+P_{22} \rho\right) \\
& +\left(e^{T} P_{11}+\rho^{T} P_{21}\right)\left[\left(A_{2}-D N H\right) e+B_{2} \eta\right. \\
& -D M \rho+\varphi(x)]+\left(e^{T} P_{12}+\rho^{T} P_{22}\right)(E \rho+G H e) \\
& -\sum_{i} 2 \lambda_{i} \eta_{i}\left(\eta_{i}-k c_{2_{i}}^{T} e\right) \\
\leq & \zeta^{T} Z_{0} \zeta+2 \beta_{d} \delta \sigma_{\max }\left(P_{11}\right)\|e\|_{2}+2 \beta_{d} \delta \sigma_{\max }\left(P_{12}\right)\|\rho\|_{2}
\end{aligned}
$$


where $\zeta=[e ; \rho ; \eta]$ and

$$
Z_{0}=\left[\begin{array}{lll}
Z_{11} & Z_{12} & Z_{13} \\
Z_{12}^{T} & Z_{22} & Z_{23} \\
Z_{13}^{T} & Z_{23}^{T} & Z_{33}
\end{array}\right] .
$$

From (17) we obtain the upper bound $\zeta^{T} Z_{0} \zeta \leq-\alpha_{1} e^{T} e-$ $\alpha_{2} \rho^{T} \rho$ and therefore

$$
\begin{aligned}
\dot{V} \leq & -\alpha_{1}\|e\|_{2}^{2}-\alpha_{2}\|\rho\|_{2}^{2}+2 \beta_{d} \delta\left[\sigma_{\max }\left(P_{11}\right)\|e\|_{2}\right. \\
& \left.+\sigma_{\max }\left(P_{12}\right)\|\rho\|_{2}\right] \\
= & \|e\|_{2}\left[-\alpha_{1}\|e\|_{2}+2 \beta_{d} \delta \sigma_{\max }\left(P_{11}\right)\right] \\
& +\|\rho\|_{2}\left[-\alpha_{2}\|\rho\|_{2}+2 \beta_{d} \delta \sigma_{\max }\left(P_{12}\right)\right] \\
\leq & -\left(\sqrt{\alpha_{1}}\|e\|_{2}-\frac{\tau_{1}}{2 \sqrt{\alpha_{1}}}\right)^{2}-\left(\sqrt{\alpha_{2}}\|\rho\|_{2}-\frac{\tau_{2}}{2 \sqrt{\alpha_{2}}}\right)^{2} \\
& +\frac{1}{4}\left(\frac{\tau_{1}^{2}}{\alpha_{1}}+\frac{\tau_{2}^{2}}{\alpha_{2}}\right) .
\end{aligned}
$$

Hence, $\dot{V}<0$ if $\forall t$

$$
\begin{aligned}
& \|e(t)\|_{2}>\eta_{1} \\
& \|\rho(t)\|_{2}>\eta_{2} .
\end{aligned}
$$

When $c_{1}$ is selected such that $\mathcal{E}_{1}^{d}$ is the smallest ellipsoid containing the balls $\mathcal{B}_{1}^{d}$ and $\mathcal{B}_{2}^{d}$ the trajectory of the error system will enter the ellipsoid $\mathcal{E}_{2}^{d}$ for every initial condition $[e(0) ; \rho(0)]$. The ellipsoid $\mathcal{E}_{2}^{d}$ is directly related to the level set of the Lyapunov function. Hence, the error system will uniformly synchronize with error bound $\sqrt{c_{3}}$ where $c_{3}$ is determined such that $\mathcal{E}_{2}^{d}$ is the smallest level set containing $\mathcal{E}_{1}^{d}$. $\mathcal{E}_{3}^{d}$ is the smallest ellipsoid containing $\mathcal{E}_{2}^{d}$, subject to the definition of these sets.

\section{ROBUST SYNTHESIS}

The design of the controller $\mathcal{C}_{s}$ is based, then, on the Theorem 1, for which one derives the following uncertainty relation between the synchronization error bound $\epsilon$ and the parameter mismatch of the systems:

$$
\frac{\epsilon}{\beta}=c_{0}
$$

where the positive real constant $c_{0}$ is equal to $2 \delta \sigma_{\max }(P) \kappa(P) / \alpha$. The interpretation of the uncertainty relation is twofold. Suppose that a larger parameter mismatch, which corresponds to an increasing $\beta$ value, suggests a larger synchronization error bound and vice-versa. Demanding a lower error bound requires a smaller parameter mismatch. An interpretation for $\beta$ follows by taking the two-norm of the functions $w_{s}(\cdot)$ and $\varphi(\cdot)$, which gives the conservative estimate

$$
\beta=\|\Delta A\|_{2}+k\left\|B_{1}\right\|_{2}\left\|C_{1}\right\|_{2}+k\left\|B_{2}\right\|_{2}\left\|C_{2}\right\|_{2} .
$$

Robust synthesis aims at minimizing the constant $c_{0}$ in the uncertainty relation such that the matrix inequality (15) is satisfied. Since $\delta$ follows from the choice of the master system, this corresponds to the following design problem for the static feedback case:

$$
\begin{aligned}
& \min _{F, P, \Lambda, \alpha} \sigma_{\max }(P) \kappa(P) / \alpha \quad \text { such that } \\
& \left\{\begin{array}{l}
Y(F, P, \Lambda, \alpha)<0 \\
P=P^{T}>0, \Lambda \geq 0 \text { and diagonal. }
\end{array}\right.
\end{aligned}
$$

For the dynamic feedback case, rather than considering the error bound as derived in Theorem 2, we will minimize the volume of the ball $\mathcal{B}_{1}^{d}$. This is meaningful as long as the condition number of the matrix $P$ remains relatively small. With respect to the ball $\mathcal{B}_{1}^{d}$, the uncertainty relation (18) has the positive real constant $c_{0}$ equal to $\eta_{1}=\left(\tau_{1} / 2 \alpha_{1}\right)+$ $(1 / 2) \sqrt{\left(\tau_{1}^{2} / \alpha_{1}^{2}\right)+\left(\tau_{2}^{2} / \alpha_{1} \alpha_{2}\right)}$ and $\beta$ corresponds to $\beta_{d}$. The design for the dynamic feedback case can be done then as follows:

$$
\begin{aligned}
\min _{E, G, M, N, P, \Lambda, \alpha_{1}, \alpha_{2}} \frac{\sigma_{\max }\left(P_{11}\right)}{\alpha_{1}}+\frac{\sigma_{\max }\left(P_{12}\right)}{\alpha_{2}} \quad \text { such that } \\
\left\{\begin{array}{l}
Z\left(E, G, M, N, P, \Lambda, \alpha_{1}, \alpha_{2}\right)<0 \\
P=P^{T}>0, \Lambda \geq 0 \text { and diagonal } \\
\kappa(P) \leq \kappa_{0}
\end{array}\right.
\end{aligned}
$$

where $\kappa_{0}$ is a user defined upper bound on the condition number of $P$.

The problems (20) and (21) are nonconvex optimization problems. Nondifferentiability might occur when the two largest eigenvalues of $Y$ or $Z$ coincide [14]. The constraint $P>0$ can be eliminated by considering the parametrization $P=Q^{T} Q$. A similar idea applies to $\Lambda$. An important observation is that the matrix inequalities, obtained for the case without parameter mismatch, are preserved (see [5], [6], and [16]) and an additional robustness criterion can be taken into account in the design procedure.

\section{EXAMPle: ChuA's CIRCUIT}

In this section we illustrate the robust synthesis method on Chua's circuit. We take the following representation for Chua's circuit:

$$
\begin{aligned}
& \dot{x}_{1}=a\left[x_{2}-h\left(x_{1}\right)\right] \\
& \dot{x}_{2}=x_{1}-x_{2}+x_{3} \\
& \dot{x}_{3}=-b x_{2}
\end{aligned}
$$

with nonlinear characteristic

$$
h\left(x_{1}\right)=m_{1} x_{1}+\frac{1}{2}\left(m_{0}-m_{1}\right)\left(\left|x_{1}+c\right|-\left|x_{1}-c\right|\right)
$$

and parameters $a=9, b=14.286, c=1 m_{0}=-1 / 7$, $m_{1}=2 / 7$ in order to obtain the double scroll attracter [3], [4], [12]. The nonlinearity $\phi\left(x_{1}\right)=1 / 2\left(\left|x_{1}+c\right|-\left|x_{1}-c\right|\right)$ (linear characteristic with saturation) belongs to sector $[0,1]$. Hence, Chua's circuit can be interpreted as the Lur'e system $\dot{x}=A x+B \phi(C x)$ where

$$
\begin{aligned}
& A=\left[\begin{array}{rrr}
-a m_{1} & a & 0 \\
1 & -1 & 1 \\
0 & -b & 0
\end{array}\right], \quad B=\left[\begin{array}{c}
-a\left(m_{0}-m_{1}\right) \\
0 \\
0
\end{array}\right], \\
& C=\left[\begin{array}{lll}
1 & 0 & 0
\end{array}\right] .
\end{aligned}
$$




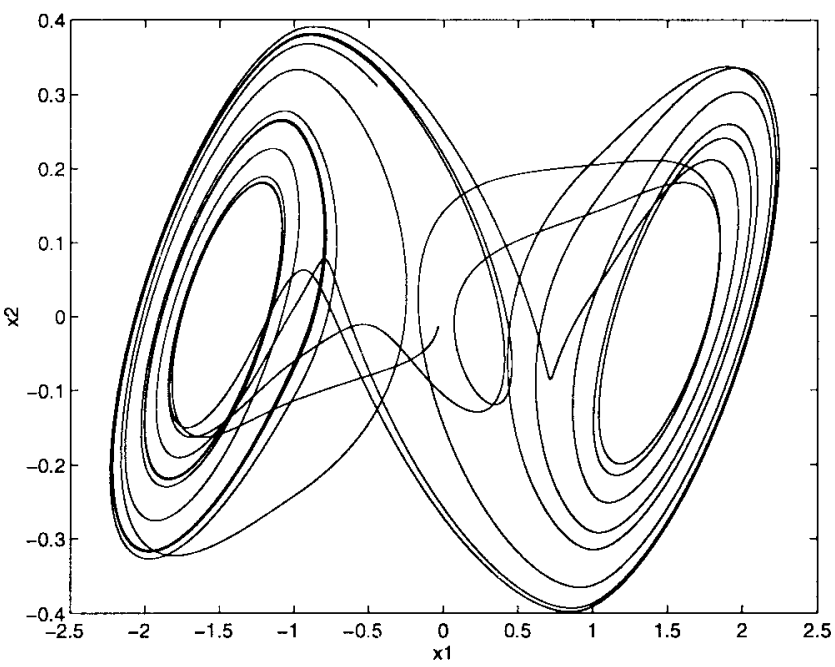

(a)

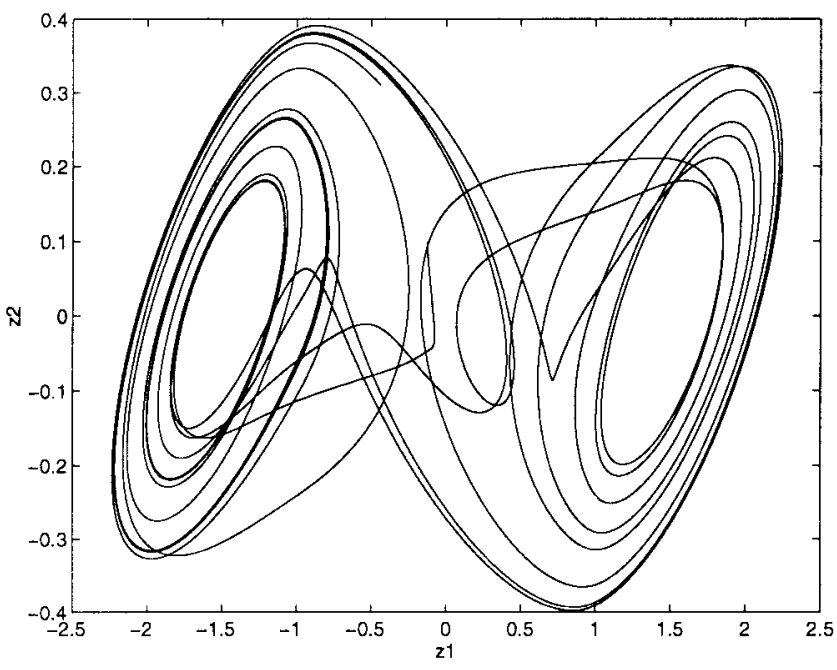

(b)

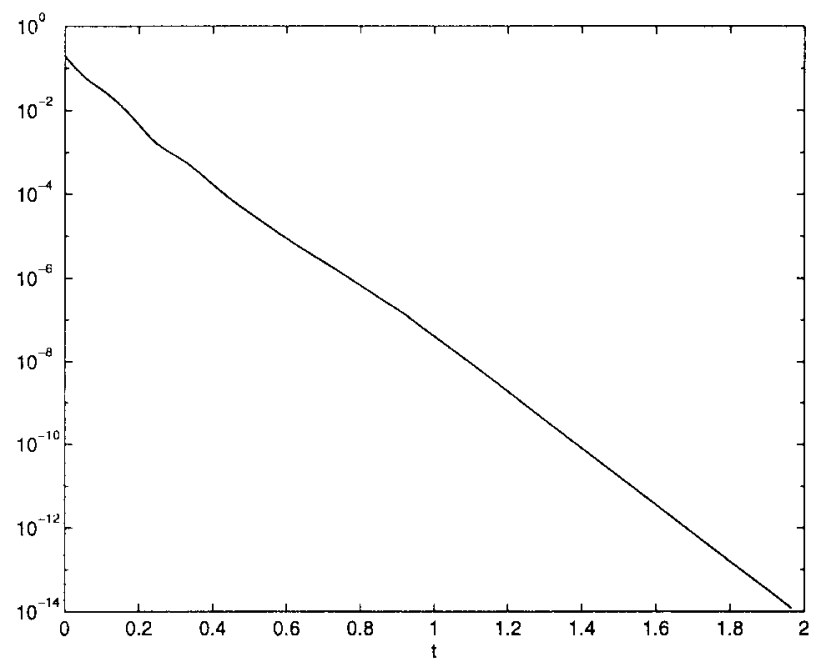

(c)

Fig. 3. Master-slave synchronization of Chua's circuits using static-state feedback. This figure shows the simulation results of the robust synthesis method in case there is no parameter mismatch. (a) Master system. (b) Slave system. (c) $\|e(t)\|_{2}$ on logarithmic scale. The synchronization error is asymptotically converging to zero.

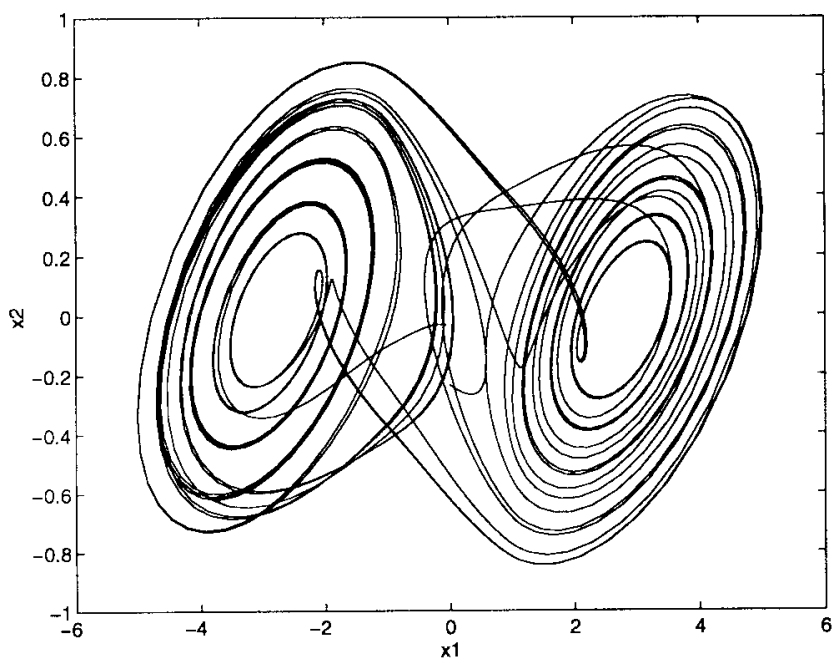

(a)

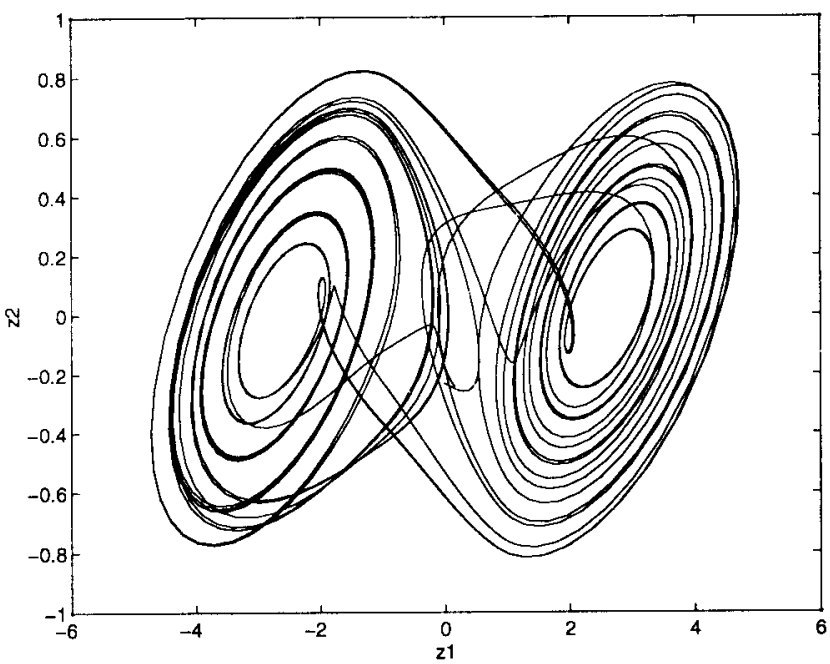

(b)

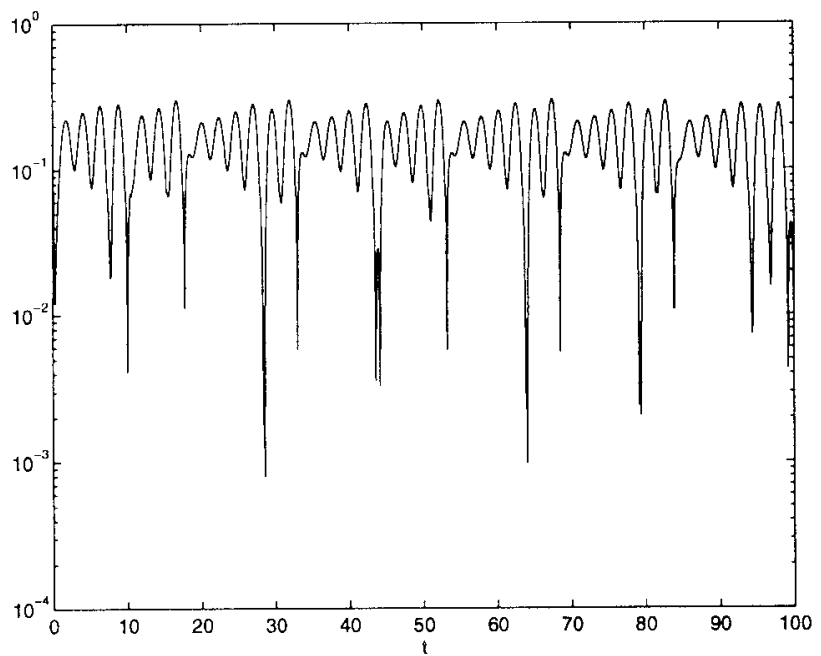

(c)

Fig. 4. Static-state feedback (continued). In this case there is parameter mismatch between the master and slave Chua's circuit. The slave system is considered to be the nominal system and behaves as the double scroll in case of a zero control input. A perturbation is considered on the $A$ matrix of the master system $\left(\delta a_{11}=1.2\right)$. The behavior of master and slave system is shown on (a) and ( b), respectively. (c) $\|e(t)\|_{2}$ on logarithmic scale. The Chua's circuits synchronize with nonzero error synchronization bound. 


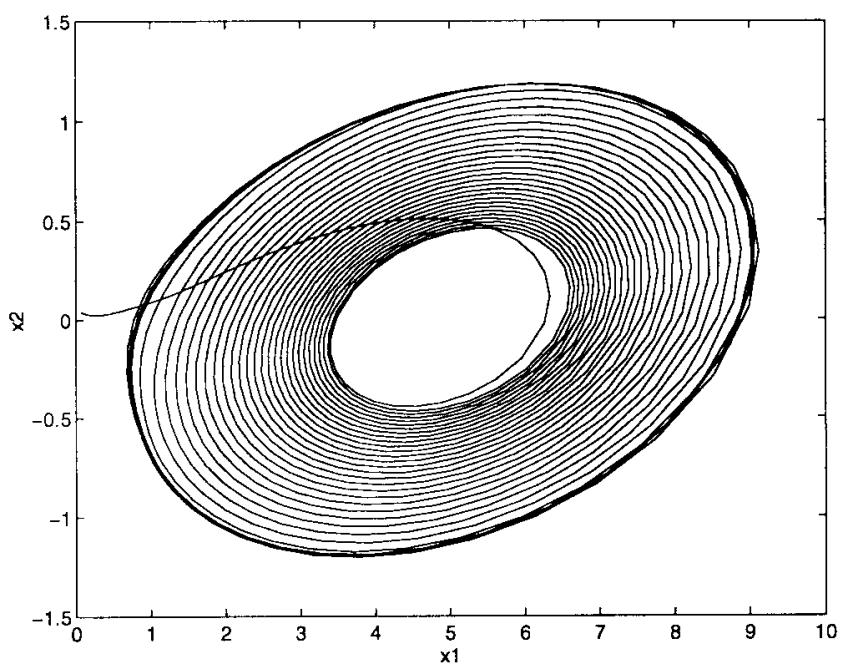

(a)

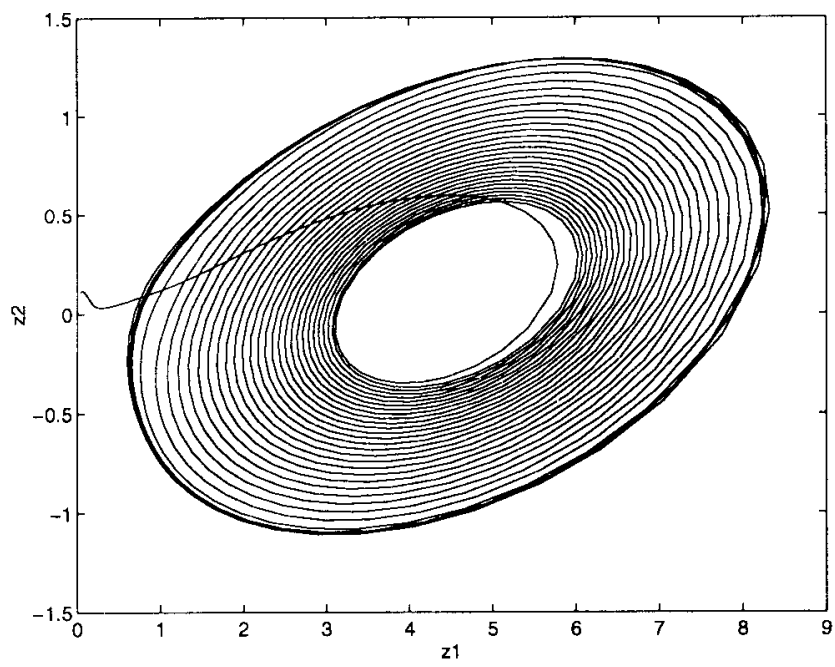

(b)

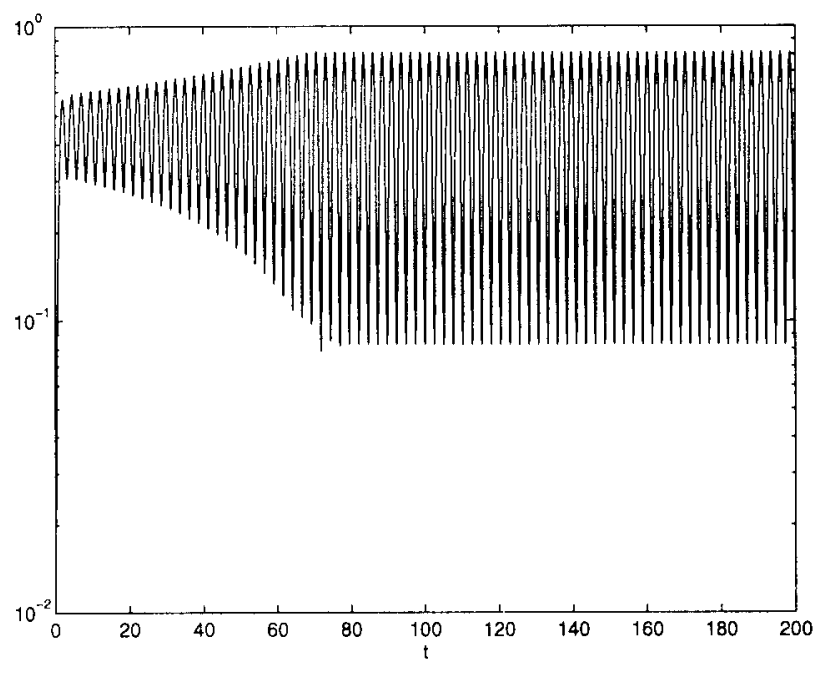

(c)

Fig. 5. Static-state feedback (continued). Similar to Fig. 2, but with perturbation $\delta a_{11}=1.8$. The master system shows limit cycle behavior, while the uncontrolled slave system behaves as the double scroll.

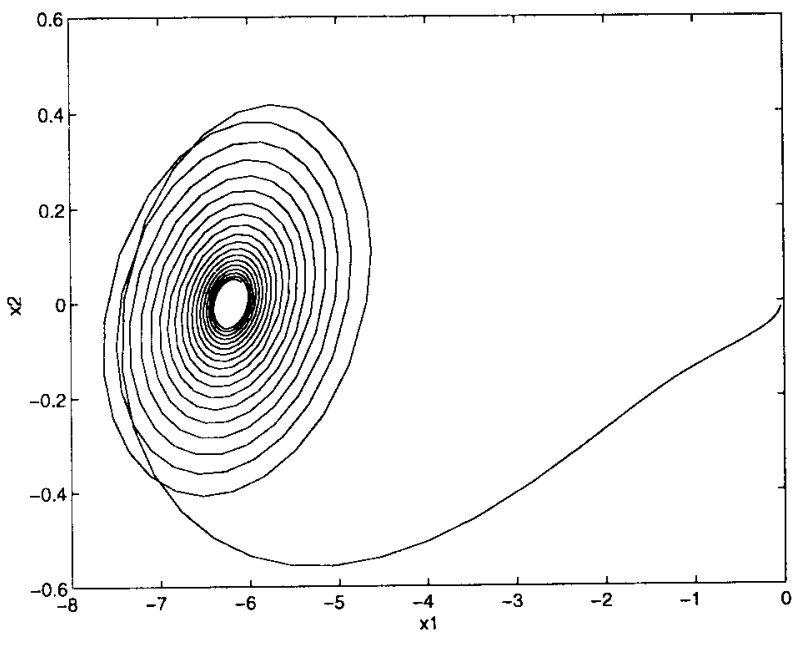

(a)

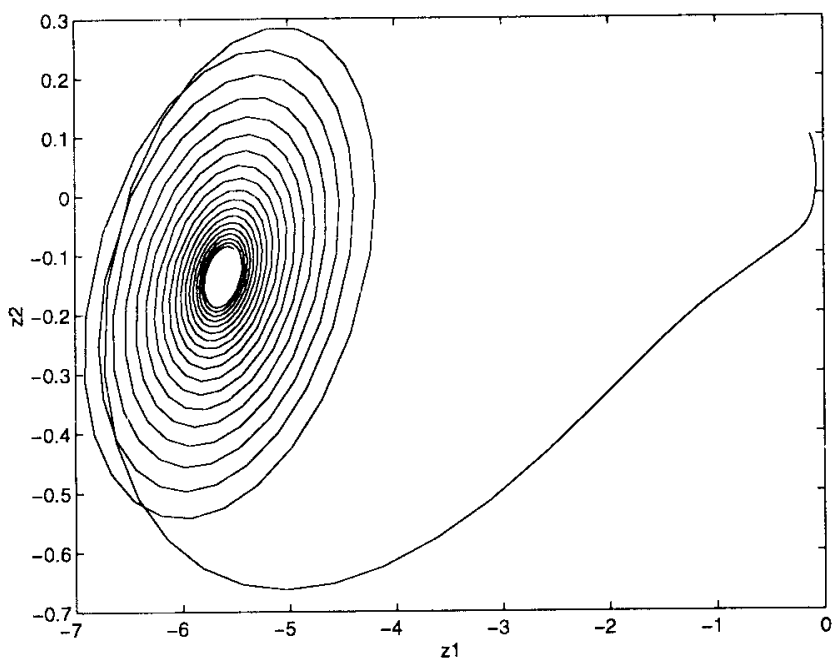

(b)

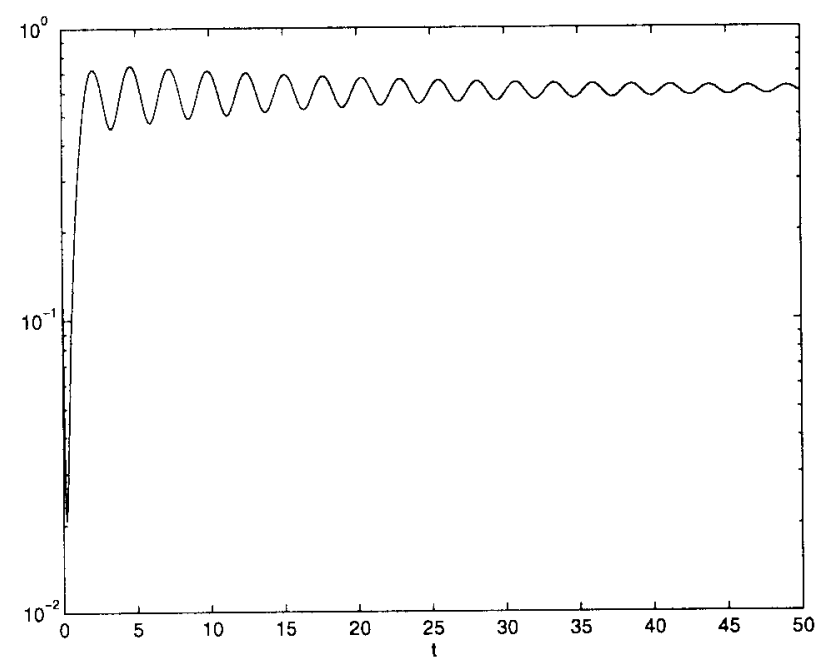

(c)

Fig. 6. Static-state feedback (continued). Similar to Fig. 2, but with perturbation $\delta a_{11}=1.95$. The master system possesses stable equilibria, while the uncontrolled slave system behaves as the double scroll. 


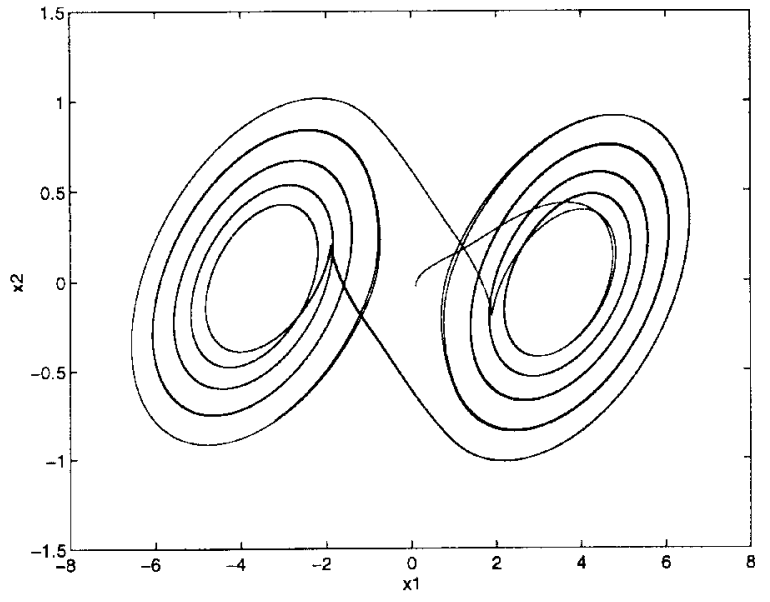

(a)

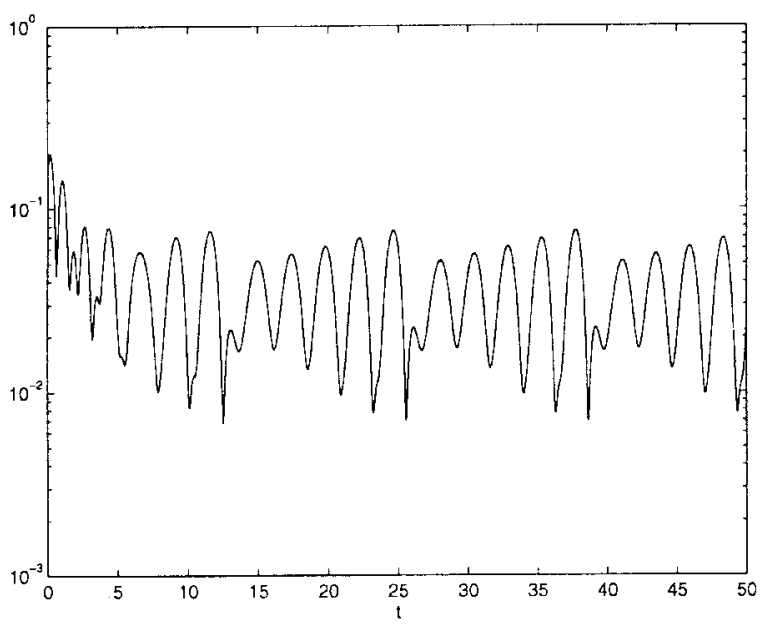

(c)

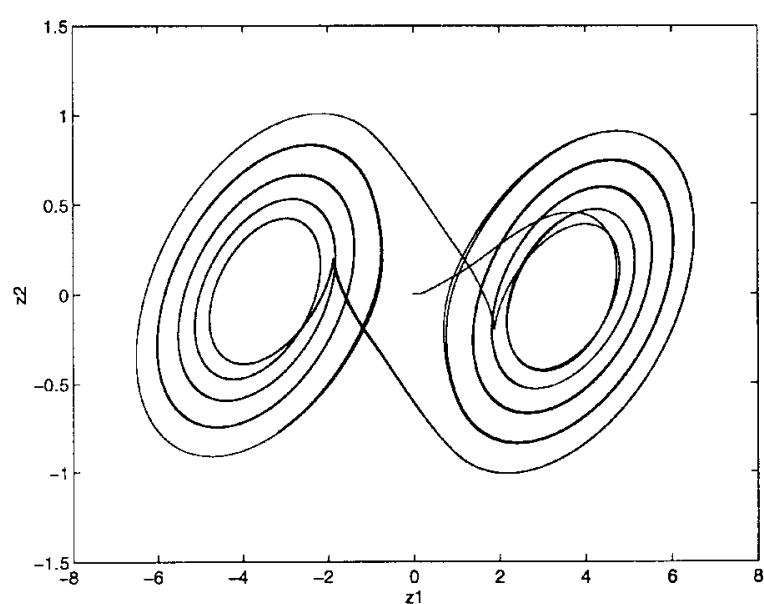

(b)

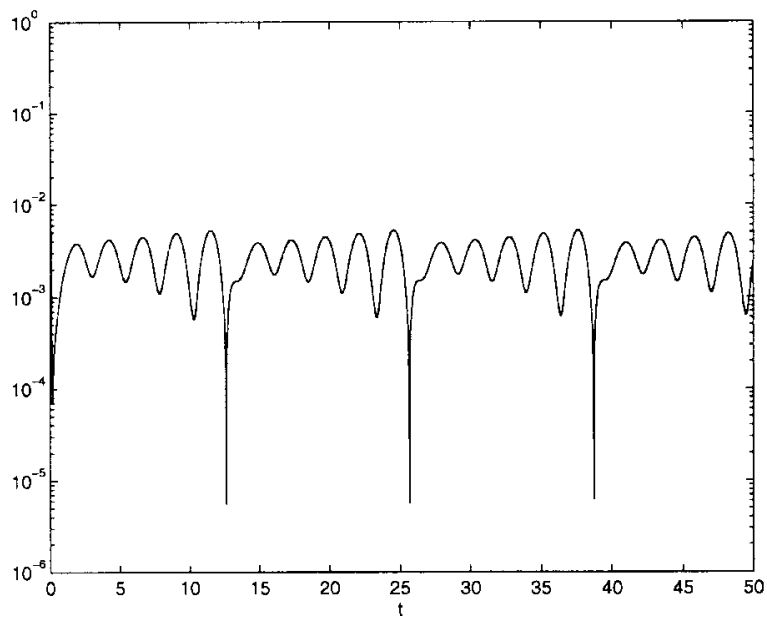

(d)

Fig. 7. Master-slave synchronization of Chua's circuits using dynamic-output feedback. A SISO second-order linear dynamic controller is considered. A perturbation of $\delta a_{11}=1.5$ is considered on the $A$ matrix of the master system. (a) Master system. (b) Slave system; (c) $\|e(t)\|_{2}$ on logarithmic scale. The Chua's circuits synchronize with nonzero error synchronization bound. (d) $\|\rho(t)\|_{2}$ on logarithmic scale.

In the sequel we consider the double scroll as the nominal system and we let it correspond to the slave system $A_{2}, B_{2}, C_{2}$.

We illustrate first the full static-state feedback case. Sequential quadratic programming [7] has been applied in order to optimize (20) using Matlab's optimization toolbox (function constr). An additional constraint on the controller parameter vector $\|F\|_{2}<20$ has been used. Instead of the inequality $Y<0$ the constraint $\lambda_{\max }(Y)+0.001<0$ has been employed where $\lambda_{\max }(\cdot)$ denotes the maximal eigenvalue of the symmetric matrix. As a starting point for the iterative procedure, a random $F$ matrix has been chosen according to a normal distribution with zero mean and variance 0.1 and $Q=I, \Lambda=0.1 I$. Simulation results for master-slave synchronization of the Chua's circuits are shown in Figs. 3-6, with perturbations on the element $a_{11}$ of the $A$ matrix of the master system. The simulation results show that it is possible to synchronize the master-slave systems with relatively small nonzero synchronization error bound, even in the case where the master system has different qualitative behavior from the uncontrolled slave system (such as limit cycles and stable equilibrium points).
In order to illustrate the dynamic-output feedback case, suppose that we measure the first state variables $x_{1}$ and $z_{1}$ only in order to synchronize the circuits and that we take a one-dimensional (1-D) control signal in order to control the slave system. This corresponds to the choice $H=\left[\begin{array}{lll}1 & 0 & 0\end{array}\right]$, $D=[1 ; 0 ; 0](l=m=1)$. We report the results here for a second-order controller with $n_{c}=2$. A two-norm constraint on the controller parameter vector $[E(:) ; G(:) ; M(:) ; N(:)](<80)$ has been used for (21), where : denotes a columnwise scanning of a matrix. Instead of the inequality $Z<0$, the constraint $\lambda_{\max }(Z)+0.001<0$ has been employed and $\kappa_{0}=20$ has been imposed. As starting point for the iterative procedure, a random controller parameter vector has been chosen according to a normal distribution with zero mean and variance 0.1 . For the matrix $Q$, a square random matrix was chosen according to the same distribution but with variance equal to three. The matrix $\Lambda$ has been initialized as $100 I$. Simulation results for master-slave synchronization of the Chua's circuits are shown in Figs. 7 and 8 for perturbations on the element $a_{11}$ of the master system. As for the static-state feedback case, it is possible to synchronize the master-slave systems with 


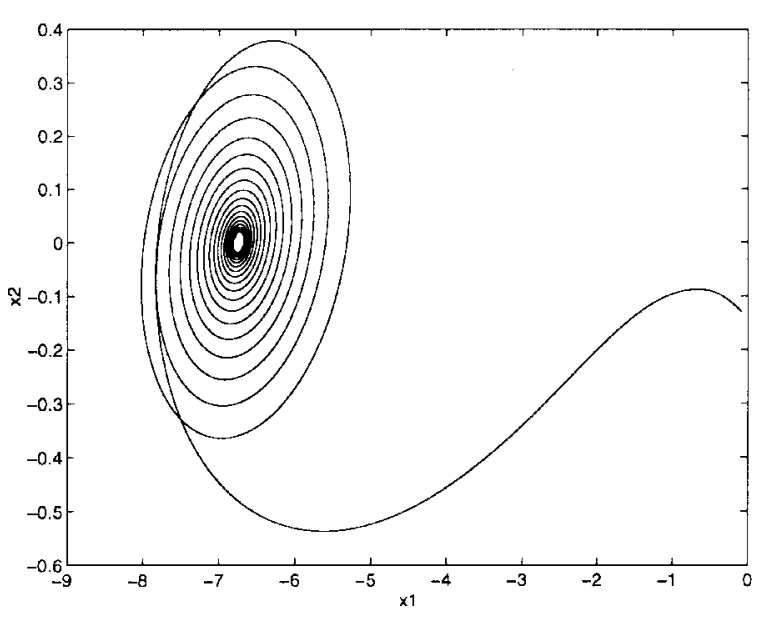

(a)

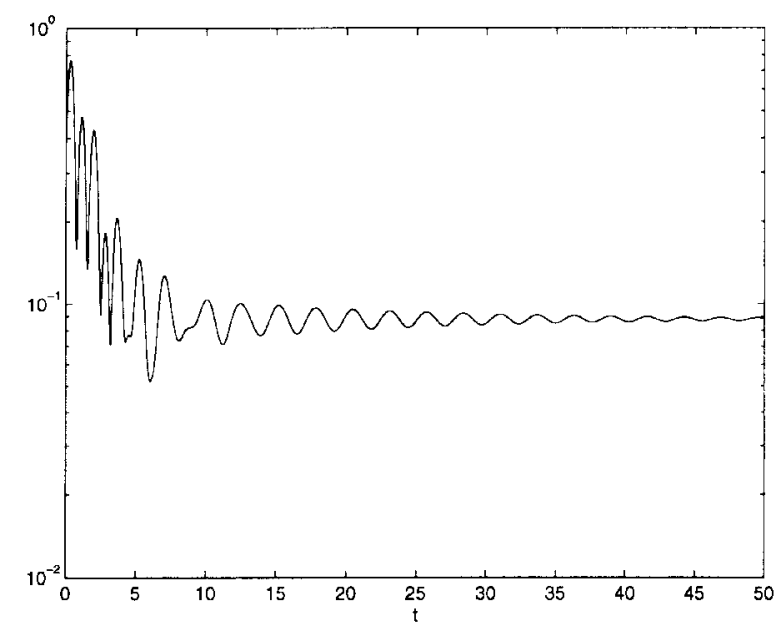

(c)

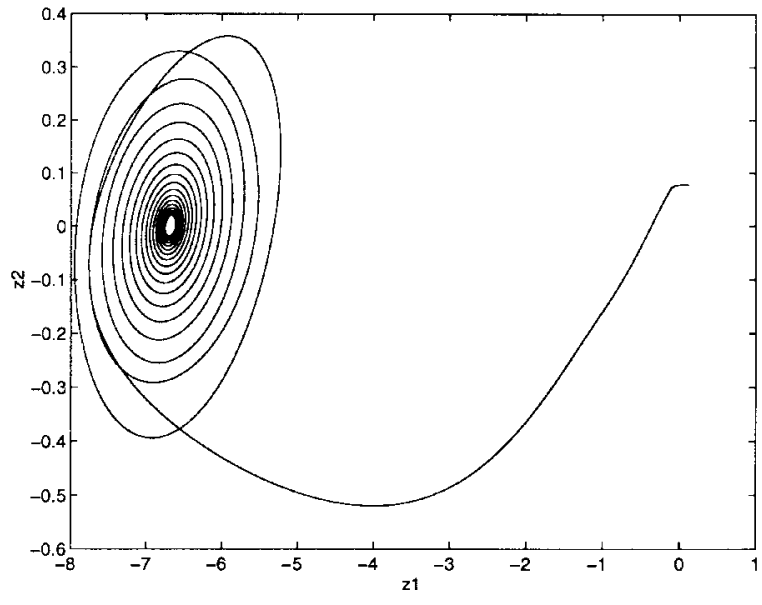

(b)

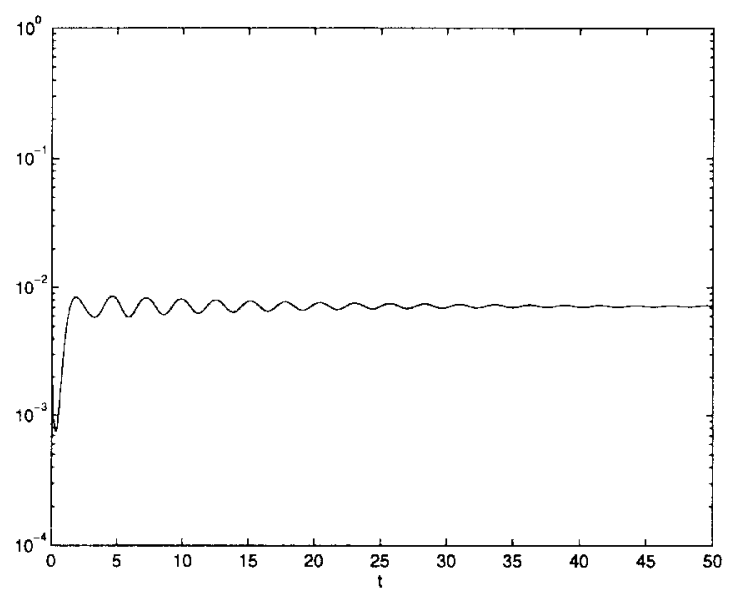

(d)

Fig. 8. Dynamic-output feedback (continued). Similar to Fig. 5, but with perturbation $\delta a_{11}=2$. The master system possesses stable equilibria, while the uncontrolled slave system behaves as the double scroll.

relatively small nonzero synchronization error bound, even in the case where the master system has different qualitative behavior from the uncontrolled slave system. All simulation results have been obtained using a Runge-Kutta integration rule with adaptive step size (ode23 in Matlab) [13].

\section{CONCLUSION}

By means of Chua's circuit we illustrated that a relatively large parameter mismatch between Lur'e systems can be allowed in order to maintain synchronization with a relatively small synchronization error bound, even in the case where the master system behaves qualitatively different from the uncontrolled slave system. These results have been obtained by deriving a robustness criterion, in addition to matrix inequalities, based upon a quadratic Lyapunov function. The matrix inequalities basically take the same form as for the case of identical Lur'e systems. The robustness criterion is based on an uncertainty relation between the synchronization error bound and the parameter mismatch. The parameter mismatch has been interpreted in terms of unstructured perturbations on the system matrices of the Lur'e systems. Both staticstate error feedback and dynamic-output error feedback have been studied. The proposed synthesis method offers a straightforward design procedure by means of solving a nonlinear optimization problem. It completes previous work on control theoretic interpretations of synchronization schemes.

\section{ACKNOWLEDGMENT}

We thank the reviewers for constructive comments.

\section{REFERENCES}

[1] S. Boyd and C. Barratt, Linear Controller Design, Limits of Performance. Englewood Cliffs, NJ: Prentice-Hall, 1991.

[2] S. Boyd, L. El Ghaoui, E. Feron, and V. Balakrishnan, Linear Matrix Inequalities in System and Control Theory, SIAM J. App. Math., vol. $15,1994$.

[3] L. O. Chua, M. Komuro, and T. Matsumoto, "The double scroll family," IEEE Trans. Circuits Syst. I, vol. 33, pp. 1072-1118, Nov. 1986.

[4] L. O. Chua, "Chua's circuit 10 years later," Int. J. Circuit Theory Appl., vol. 22, pp. 279-305, 1994.

[5] P. F. Curran and L. O. Chua, "Absolute stability theory and the synchronization problem," Int. J. Bifurcation Chaos, vol. 7, no. 6, pp. 1375-1382, 1997.

[6] P. F. Curran, J. A. K. Suykens, and L. O. Chua, "Absolute stability theory and master-slave synchronization," Int. J. Bifurcation and Chaos, vol. 7, no. 12, 1997.

[7] R. Fletcher, Practical Methods of Optimization. New York: Wile, 1987. 
[8] M. Hasler, "Synchronization principles and applications," in Proc. Circuits and Systems: Tutorials IEEE-ISCAS '94, 1994, pp. 314-326.

[9] H. K. Khalil, Nonlinear Systems. New York: Macmillan, 1992.

[10] J. P. LaSalle and S. Lefschetz, Stability by Liapunov's Direct Method with Applications. New York: Academic, 1961.

[11] J. M. Maciejowski, Multivariable Feedback Design. Reading, MA: Addison-Wesley, 1989.

[12] R. N. Madan, Ed., Chua's Circuit: A Paradigm for Chaos. Signapore: World Scientific, 1993

[13] T. S. Parker and L. O. Chua, Practical Numerical Algorithms for Chaotic Systems. New York: Springer-Verlag, 1989.

[14] E. Polak and Y. Wardi, "Nondifferentiable optimization algorithm for designing control systems having singular value inequalities," Automatica, vol. 18 , no. 3, pp. 267-283, 1982.

[15] J. A. K. Suykens, J. P. L. Vandewalle, and B. L. R. De Moor, Artificial Neural Networks for Modeling and Control of Non-Linear Systems. Boston, MA: Kluwer, 1996.

[16] J. A. K. Suykens, P. F. Curran, and L. O. Chua, "Master-slave synchronization using dynamic output feedback," Int. J. Bifurcation Chaos, vol. 7, no. 3, pp. 671-679, 1997.

[17] J. A. K. Suykens, J. Vandewalle, and L. O. Chua, "Nonlinear $H_{\infty}$ synchronization of chaotic Lur'e systems," Int. J. Bifurcation Chaos, vol. 7, no. 6, pp. 1323-1335, 1997.

[18] J. A. K. Suykens, P. F. Curran, T. Yang, J. Vandewalle, and L. O. Chua, "Nonlinear $H_{\infty}$ synchronization of Lur'e systems: Dynamic output feedback case," IEEE Trans. Circuits Syst. I, vol. 44, pp. 1089-1092, Nov. 1997.

[19] J. A. K. Suykens, P. F. Curran, J. Vandewalle, and L. O. Chua, "Robust nonlinear $H_{\infty}$ synchronization of chaotic Lur'e systems," IEEE Trans. Circuits Syst. I, vol. 44, pp. 891-904, Oct. 1997.

[20] M. Vidyasagar, Nonlinear Systems Analysis. Englewood Cliffs, NJ: Prentice-Hall, 1993.
[21] C. W. Wu and L. O. Chua, "A unified framework for synchronization and control of dynamical systems," Int. J. Bifurcation Chaos, vol. 4, no. 4, pp. 979-989, 1994.

Johan A. K. Suykens was born in Willebroek, Belgium, on May 18, 1966 $\mathrm{He}$ received the degree in electro-mechanical engineering and the $\mathrm{Ph} . \mathrm{D}$ degree in applied sciences from the Katholieke Universiteit Leuven, in 1989 and 1995, respectively.

In 1996, he was a Visiting Postdoctoral Researcher at the University of California, Berkeley. At present he is a Postdoctoral Researcher with the Fund for Scientific Research, FWO, Flanders. His research interests are in the areas of the theory and application of nonlinear systems and neural network. $\mathrm{He}$ is Author of Artificial Neural Networks for Modeling and Control of Non-linear Systems and Editor of the book Nonlinear Modeling: Advanced Black-Box Techniques."

Dr. Suykens is currently serving as Associate Editor for the IEEE Transactions on Circuits and Systems-I and for the IEEE Transactions on NeURAL NeTwORKS.

Paul F. Curran, for a photograph and biography, see p. 10 of the October 1997 issue of this Transactions.

Leon O. Chua, for a photograph and biography, see p. 10 of the October 1997 issue of this TRANSACTIONS. 